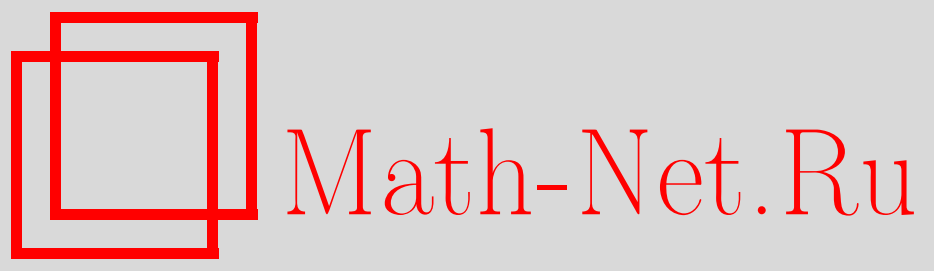

С. Л. Яковлев, Е. А. Яревский, Н. О. Эландер, А. К. Беляев, Об асимптотическом решении многоканальной задачи рассеяния с неадиабатической связью каналов, TMФ, 2018, том 195, номер 3, 437-450

DOI: https://doi.org/10.4213/tmf9451

Использование Общероссийского математического портала Math-Net.Ru подразумевает, что вы прочитали и согласны с пользовательским соглашением http: //www . mathnet.ru/rus/agreement

Параметры загрузки:

IP : 54.81 .137 .203

26 апреля 2023 г., $17: 57: 11$

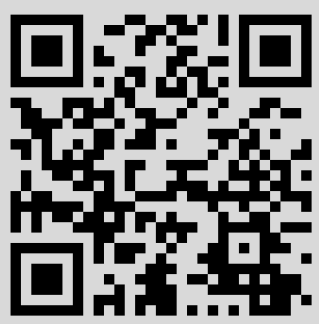




\title{
ОБ АСИМПТОТИЧЕСКОМ РЕШЕНИИ МНОГОКАНАЛЬНОЙ ЗАДАЧИ РАССЕЯНИЯ С НЕАДИАБАТИЧЕСКОЙ СВЯЗЬЮ КАНАЛОВ
}

\begin{abstract}
Рассмотрена многоканальная задача рассеяния в адиабатическом представлении. Предполагается, что матрица неадиабатической связи каналов имеет нетривиальное значение на больших межъядерных расстояниях. Построены асимптотические решения на больших межъядерных расстояниях. Показано, что эти асимптотические решения вплоть до первого порядка теории возмущений идентичны асимптотическим решениям подхода перепроецирования, который был предложен ранее в качестве средства для решения проблемы трансляции электронов в контексте метода Борна-Оппенгеймера.
\end{abstract}

Ключевые слова: многоканальное рассеяние, асимптотическое решение задачи рассеяния, адиабатическое приближение.

DOI: https://doi.org/10.4213/tmf9451

\section{1. ВВЕДЕНИЕ}

1.1. История вопроса. Адиабатический подход является одним из наиболее широко используемых методов для теоретического изучения низкоэнергетической квантовой задачи столкновений в атомной и молекулярной физике [1]. В контексте задачи двухатомных столкновений он состоит в использовании собственных функций электронного гамильтониана в качестве базиса для разложения полной волновой функции, которое затем ведет к результирующим многоканальным уравнениям. Одним из главных приближений в рамках этого подхода является приближение

Работа С. Л. Яковлева и Е.А. Яревского поддержана РФФИ (грант № 18-0200492_a). Работа Н. О. Эландера поддержана Carl Trygger Foundation. Работа А. К. Беляева поддержана РФФИ (грант № 16-03-00149).

\footnotetext{
* Санкт-Петербургский государственный университет, Санкт-Петербург, Россия.
} E-mail: s.yakovlev@spbu.ru

${ }^{\dagger}$ Stockholm University, Stockholm, Sweden

${ }^{\ddagger}$ Российский государственный педагогический университет им. А. И. Герцена, Санкт-Петербург, Россия 
Борна-Оппенгеймера [2], которое соответствует пренебрежению всеми неадиабатическими связями каналов. Адиабатическое представление является полным, если все неадиабатические связи каналов сохраняются в формализме, и, следовательно, оно в принципе дает точное решение уравнения Шредингера. Главная с вычислительной точки зрения трудность решения задачи рассеяния в адиабатическом приближении связана с наличием сильной неадиабатической связи каналов. Критичным образом это может проявиться в районе (запрещенных) пересечений электронных термов энергии, которые, как правило, наблюдаются при конечных межъядерных расстояниях. Одним из методов, позволяющих преодолеть эту трудность, является переформулировка задачи посредством перехода в диабатическое представление или в более гибкое диабатическое представление с расщеплением (см., например, работы [3], [4] и ссылки в них). Другой источник нетривиальной неадиабатической связи каналов связан с так называемой проблемой молекулярных состояний (иногда называемой проблемой трансляции электронов), которая проявляется в формализме как ненулевой предел матрицы неадиабатической связи каналов, когда межъядерное расстояние стремится к бесконечности [5]-[10]. Выяснено (см. статьи [9]-[12]), что физической причиной неубывания элементов матрицы асимптотической связи каналов является то, что адиабатический подход основывается на представлении молекулярных состояний и тем самым - на молекулярных координатах (а именно, на молекулярных координатах Якоби), в которых местоположение электронов отсчитывается от центра масс атомного ядра. Проблема состоит в том, что молекулярные координаты, которые используются для описания молекулярных состояний сталкивающихся комплексов с фиксированными ядрами при малых и средних расстояниях, не пригодны для описания свободных атомов в асимптотической области. Разница в описании возникает из-за того, что векторы межатомных расстояний, соединяющие центры масс сталкивающихся атомов, не совпадают с векторами межъядерных расстояний, соединяющих центры масс соответствующих ядер. Это обстоятельство ведет к возникновению ненулевых матричных элементов матрицы асимптотической связи каналов, вычисленной в молекулярном представлении. Таким образом, ненулевая асимптотическая связь каналов в многоканальных уравнениях является фундаментальным свойством стандартного подхода Борна-Оппенгеймера. Это свойство приводит к тому, что простая асимптотическая форма Борна-Оппенгеймера для волновой функции рассеяния в адиабатическом приближении более не справедлива.

Асимптотический вид решения в специальном случае трехчастичной задачи рассеяния в адиабатическом представлении изучался в работах [13]-[15] и статье [16]. Достаточно общий подход к задаче многоканального рассеяния с потенциалами, зависящими от скорости, был предложен в работе [17]. Этот метод нашел широкое применение в расчетах мезоатомных и мезомолекулярных процессов [18]. Один из успешных практических методов для построения подходящей асимптотической формы волновой функции в адиабатическом представлении реализуется с помощью процедуры перепроецирования [9]-[12]. Эта процедура использует физически обоснованную асимптотическую форму полной волновой функции, которая представляется в координатах Якоби, специфицированными каналами, с помощью собственных состояний невзаимодействующих атомов (см., например, работу [19]). Асимптотическая форма волновой функции в адиабатическом представлении затем вычисляется 
с помощью перепроецирования этой полной волновой функции на адибатический (молекулярный) базис. Хотя эта процедура и является хорошо обоснованной, однако по отношению к собственно адиабатическому представлению она требует внешней информации об асимптотической форме полной волновой функции. С другой стороны, полное адиабатическое представление должно автоматически генерировать корректную форму волновой функции в адиабатическом представлении, а асимптотическая волновая функция должна быть построена из асимптотических решений адиабатических связанных многоканальных уравнений в области больших межъядерных расстояний. Один из примеров такого подхода дан в статье [17]. Однако такой метод построения асимптотической волновой функции, которая играет роль граничных условий в адиабатическом представлении, требует дальнейшего развития. Это послужило мотивацией для настоящей работы. Мы строим асимптотические решения для адиабатических связанных уравнений, используя только такое общее свойство матрицы связи каналов, как антисимметричность.

Работа организована следующим образом. В п. 1.2 дана постановка задачи. Двухканальная модель рассмотрена в разделе 2, так как она дает возможность продемонстрировать явное решение задачи со всеми необходимыми деталями. В разделе 3 мы рассматриваем общую ситуацию, когда асимптотическая матрица неадиабатической связи каналов связывает произвольное количество каналов $N$. Раздел 4 содержит заключение. Доказательство технического утверждения о корнях специального полинома с матричными коэффициентами, возникающего в формализме, дано в приложении.

1.2. Многоканальные адиабатические уравнения и асимптотические состояния Борна-Оппенгеймера. Рассматривается задача рассеяния для системы уравнений

$$
\left[-\frac{d^{2}}{d r^{2}}+\frac{\ell(\ell+1)}{r^{2}}+V_{j}(r)-E\right] F_{j}(r)=\sum_{m \geqslant 1}\left[2 P_{j m}(r) \frac{d}{d r}+W_{j m}(r)\right] F_{m}(r),
$$

определенной для целых $\ell \geqslant 0$ и вещественных неотрицательных $r, 0 \leqslant r<\infty$. Коэффициенты $V_{j}(r), P_{j m}(r)$ и $W_{j m}(r)$ определены ниже. Для корректной формулировки задачи систему (1) следует дополнить соответствующими граничными условиями. В то время как граничное условие при $r=0$ является естественным: $F_{j}(0)=0$, асимптотический при $r \rightarrow \infty$ вид $F_{j}(r)$ далек от тривиального и зависит от степени убывания коэффициентов $V_{j}(r), P_{j m}(r)$ и $W_{j m}(r)$, когда $r \rightarrow \infty$.

Система уравнений (1) представляет собой уравнение Шредингера для системы двух атомов при использовании так называемого адиабатического разложения [1] для полной волновой функции $\Psi$ системы. Молекулярный базис $\phi_{k}$ генерирует разложение полной волновой функции

$$
\Psi(\mathbf{r}, \boldsymbol{\xi})=Y_{\ell}^{\mu_{\ell}}(\hat{r}) \sum_{m \geqslant 1} \frac{F_{m}(r)}{r} \phi_{m}(\boldsymbol{\xi}, r),
$$

где $Y_{\ell}^{\mu_{\ell}}$ - стандартные сферические функции, для которых $\ell$ и $\mu_{\ell}$ обозначают полный угловой момент и его проекцию, $\mathbf{r}$ обозначает относительный вектор межъядерного расстояния, единичный вектор $\hat{r}$ дается выражением $\mathbf{r} / r$, в котором $r=|\mathbf{r}|$, а $\boldsymbol{\xi}$ представляет электронные степени свободы. Предыдущее разложение (2) подразумевает 
следующую форму двухатомного гамильтониана:

$$
H=-\frac{\hbar^{2}}{2 M} \nabla_{\mathbf{r}}^{2}+h(\mathbf{r}, \boldsymbol{\xi})
$$

где $M$ обозначает приведенную массу ядер. Гамильтониан $h(\mathbf{r}, \boldsymbol{\xi})$ содержит все потенциалы взаимодействия и управляет динамикой электронов в поле "замороженных" ядер. Молекулярный базис $\phi_{m}(\boldsymbol{\xi}, r)$ тогда формируется из собственных функций гамильтониана $h(\mathbf{r}, \boldsymbol{\xi})$ :

$$
h(\mathbf{r}, \boldsymbol{\xi}) \phi_{m}(\boldsymbol{\xi}, r)=\lambda_{m}(r) \phi_{m}(\boldsymbol{\xi}, r) .
$$

Собственные функции удовлетворяют условиям ортогональности и полноты

$$
\left\langle\phi_{j} \mid \phi_{m}\right\rangle=\delta_{j m}, \quad \sum_{m \geqslant 1}\left|\phi_{m}(\boldsymbol{\xi}, r)\right\rangle\left\langle\phi _ { m } \left(\boldsymbol{\xi}^{\prime}, r \mid=\delta\left(\boldsymbol{\xi}-\boldsymbol{\xi}^{\prime}\right)\right.\right.
$$

где $\delta_{j m}$ - символ Кронекера, а $\delta\left(\boldsymbol{\xi}-\boldsymbol{\xi}^{\prime}\right)$ - дельта-функция. Относительный вектор межъядерного расстояния здесь служит параметром. На протяжении работы предполагается, что собственные функции $\phi_{m}(\boldsymbol{\xi}, r)$ и собственные значения $\lambda_{m}(r)$ зависят от модуля $r=|\mathbf{r}|$ вектора $\mathbf{r}$. Это свойство является весьма общим во многих приложениях [8] и соответствует, например, случаю, когда только молекулярные $\Sigma$-состояния вносят вклад в задачу. Это предположение можно приспособить и к более общей ситуации, которая включает в себя ряд небольших усложнений в матрице связи каналов [10]. Наконец, предполагается, что элементы молекулярного базиса $\phi_{j}$ выбраны вещественными, что всегда возможно, так как гамильтониан $h(\mathbf{r}, \boldsymbol{\xi})$ эрмитов.

Матрицы в уравнениях (1) теперь можно определить в терминах молекулярного базиса следующими уравнениями:

$$
\begin{gathered}
V_{j}(r)=\frac{2 M}{\hbar^{2}} \lambda_{j}(r), \\
P_{j m}(r)=\frac{2 M}{\hbar^{2}}\left\langle\phi_{j}\left|\frac{\partial}{\partial r}\right| \phi_{m}\right\rangle, \quad W_{j m}(r)=\frac{2 M}{\hbar^{2}}\left\langle\phi_{j}\left|\frac{\partial^{2}}{\partial r^{2}}\right| \phi_{m}\right\rangle .
\end{gathered}
$$

Здесь скобки в матричных элементах означают интегрирование по электронным степеням свободы $\boldsymbol{\xi}$. Полнота базиса ведет к хорошо известному соотношению между матрицами $P_{j m}(r)$ и $W_{j m}(r)$

$$
W_{j m}(r)=\sum_{p \geqslant 1} P_{j p}(r) P_{p m}(r)+\frac{d}{d r} P_{j m}(r) .
$$

Так как собственные функции $\phi_{j}$ вещественны, матрица $P_{j m}(r)$ является антисимметричной, т. е. $P_{j m}(r)=-P_{m j}(r)$, и, следовательно, она является внедиагональной, $P_{j j}(r)=0$. Постоянная $E$ в системе (1) представляет собой приведенную полную энергию в сталкивающейся системе.

Асимптотическое поведение потенциалов $V_{j}(r)$ и матриц $P_{j m}(r)$ является существенным для асимптотического анализа решений уравнений (1). Асимптотические значения $V_{j}(r)$ определяют асимптотические пороги $\epsilon_{j}$ :

$$
\epsilon_{j}=\lim _{r \rightarrow \infty} V_{j}(r) .
$$


В настоящей работе мы предполагаем, что потенциалы $V_{j}(r)$ являются короткодействующими, т. е.

$$
\lim _{r \rightarrow \infty} r^{1+\delta}\left[V_{j}(r)-\epsilon_{j}\right]=0
$$

для $\delta>0$. Матрица $P_{j m}(r)$ определяет асимптотическую связь $a_{j m}$ формулой

$$
a_{j m}=\lim _{r \rightarrow \infty} P_{j m}(r)
$$

Как и для потенциалов, требуется выполнение условия

$$
\lim _{r \rightarrow \infty} r^{1+\delta}\left[P_{j m}(r)-a_{j m}\right]=0
$$

для $\delta>0$. Эти условия “короткодействия" ведут к следующим асимптотическим свойствам матриц в уравнениях (1):

$$
\begin{aligned}
V_{j}(r) & \sim \epsilon_{j}+O\left(r^{-(1+\delta)}\right), \\
P_{j m}(r) & \sim a_{j m}+O\left(r^{-(1+\delta)}\right), \\
W_{j m}(r) & \sim \sum_{p \geqslant 1} a_{j p} a_{p m}+O\left(r^{-(1+\delta)}\right) .
\end{aligned}
$$

На основании этих свойств уравнения (1) можно преобразовать к виду

$$
\left[-\frac{d^{2}}{d r^{2}}+\epsilon_{j}-E\right] F_{j}(r)=\sum_{m \geqslant 1}\left[2 a_{j m} \frac{d}{d r}+\sum_{p \geqslant 1} a_{j p} a_{p m}\right] F_{m}(r)+Q_{j}(r, F) .
$$

В этом уравнении все члены порядка $O\left(r^{-(1+\delta)}\right)$ и менее обозначены $Q_{j}(r, F)$. Из формальной теории рассеяния [20] следует, что члены порядка $O\left(r^{-(1+\delta)}\right)$ не влияют на форму асимптотического поведения решения при $r \rightarrow \infty$. Поэтому в рассматриваемой задаче изучение асимптотики решения уравнения (16) в предположении, что $Q_{j}(r, F)=0$, дает корректную асимптотическую форму решения уравнения (1).

В зависимости от значения асимптотической связи $a_{j m}$ следует различать два случая: 1) $a_{j m}=0$ для всех $j, m$ и 2) существует номер $N$ такой, что $a_{j m} \neq 0$ для $j, m \leqslant N$ и $a_{j m}=0$, если $j>N$ или $m>N$.

Первый случай в определенном смысле является общепринятым и соответствует асимптотическим состояниям типа Борна-Оппенгеймера [21]. По существу, благодаря условиям (10) и (12) система уравнений в (16) становится расцепленной в пределе $r \rightarrow \infty$ и принимает форму

$$
\left[-\frac{d^{2}}{d r^{2}}+\epsilon_{j}-E\right] F_{j}(r)=0
$$

Два линейно независимых решения (17) даются формулами

$$
F_{j}^{ \pm}\left(r, k_{j}\right)=e^{ \pm i k_{j} r}
$$

где $i=\sqrt{-1}$ и волновые числа каналов $k_{j}$ имеют вид

$$
k_{j}=\sqrt{E-\epsilon_{j}} \geqslant 0 .
$$


Эти решения предоставляют базис для асимптотического решения уравнений (1) при $r \rightarrow \infty$

$$
F_{j}(r) \sim k_{j}^{-1 / 2}\left[b_{j}^{+} F_{j}^{+}\left(r, k_{j}\right)+b_{j}^{-} F_{j}^{-}\left(r, k_{j}\right)\right] .
$$

Матрицу рассеяния теперь можно определить как матрицу преобразования между амплитудами сходящихся и расходящихся волн:

$$
(-1)^{\ell+1} b_{j}^{+}=\sum_{m} S_{j m} b_{m}^{-}
$$

Второй случай является намного более сложным и изучается в последующих разделах.

\section{2. ДВУХКАНАЛЬНАЯ МОДЕЛЬ}

Двухканальная модель рассматривается как первый шаг в решении задачи построения асимптотической формы решения уравнения (1). В матричной форме эта модель записывается в виде

$$
\left\{-\mathbf{I}\left[\frac{d^{2}}{d r^{2}}-\frac{\ell(\ell+1)}{r^{2}}\right]-2 \mathbf{P}(r) \frac{d}{d r}-\mathbf{W}(r)+\mathbf{V}(r)-\mathbf{I} E\right\} \mathbf{F}(r)=0,
$$

где $\mathbf{F}=\left\{F_{1}(r), F_{2}(r)\right\}^{\mathrm{T}}$, матрицы $\mathbf{I}, \mathbf{P}$, и $\mathbf{V}$ определены следующим образом:

$$
\mathbf{I}=\left[\begin{array}{ll}
1 & 0 \\
0 & 1
\end{array}\right], \quad \mathbf{P}(r)=\left[\begin{array}{cc}
0 & p(r) \\
-p(r) & 0
\end{array}\right], \quad \mathbf{V}=\left[\begin{array}{cc}
V_{1}(r) & 0 \\
0 & V_{2}(r)
\end{array}\right]
$$

a

$$
\mathbf{W}(r)=\mathbf{P}^{2}(r)+\frac{d}{d r} \mathbf{P}(r) .
$$

Так как асимптотическая связь каналов должна быть нетривиальной при $r \rightarrow \infty$, то мы имеем соотношение

$$
\lim _{r \rightarrow \infty} p(r)=a \neq 0 .
$$

В отличие от системы (17), система уравнений (22) остается связанной при $r \rightarrow \infty$ и асимптотически принимает вид

$$
\left(-\mathbf{I} \frac{d^{2}}{d r^{2}}-2 \mathbf{A} \frac{d}{d r}-\mathbf{A}^{2}-\mathbf{K}^{2}\right) \boldsymbol{\Phi}(r)=0 .
$$

Здесь $\boldsymbol{\Phi}=\left\{\Phi_{1}(r), \Phi_{2}(r)\right\}^{\mathrm{T}}$, а $\mathbf{A}$ и $\mathbf{K}^{2}$ являются постоянными матрицами размера $2 \times 2$, определяемыми равенствами

$$
\mathbf{A}=\left[\begin{array}{rr}
0 & a \\
-a & 0
\end{array}\right], \quad \mathbf{K}^{2}=\left[\begin{array}{cc}
k_{1}^{2} & 0 \\
0 & k_{2}^{2}
\end{array}\right]
$$

Следующим шагом является построение линейно независимых решений уравнения (25). Эти решения мы представим следующим выражением для компонент:

$$
\Phi_{j}(r)=c_{j} e^{i q r}, \quad j=1,2,
$$


в которых $q$ и $c_{j}$ следует определить так, чтобы вектор-функция $\boldsymbol{\Phi}(r)$ удовлетворяла уравнению $(25)$. С компонентами $\Phi_{j}$, представленными в этой форме, система дифференциальных уравнений (25) сводится к следующей системе линейных алгебраических уравнений:

$$
\left(q^{2}-k_{1}^{2}+a^{2}\right) c_{1}+i 2 a q c_{2}=0, \quad\left(q^{2}-k_{2}^{2}+a^{2}\right) c_{2}-i 2 a q c_{1}=0 .
$$

Параметр $q$ следует теперь определить из условия, что эта система однородных уравнений имеет нетривиальное решение. Это может быть достигнуто, если детерминант системы (28) обратится в ноль, что ведет к следующему биквадратному уравнению:

$$
q^{4}-\left(k_{1}^{2}+k_{2}^{2}+2 a^{2}\right) q^{2}+\left(k_{1}^{2}-a^{2}\right)\left(k_{2}^{2}-a^{2}\right)=0 .
$$

Четыре решение уравнения (29) даются формулами

$$
\begin{aligned}
& q_{1}^{ \pm}= \pm \frac{1}{\sqrt{2}} \sqrt{k_{1}^{2}+k_{2}^{2}+2 a^{2}+\sqrt{\left(k_{1}^{2}+k_{2}^{2}+2 a^{2}\right)^{2}-4\left(k_{1}^{2}-a^{2}\right)\left(k_{2}^{2}-a^{2}\right)},} \\
& q_{2}^{ \pm}= \pm \frac{1}{\sqrt{2}} \sqrt{k_{1}^{2}+k_{2}^{2}+2 a^{2}-\sqrt{\left(k_{1}^{2}+k_{2}^{2}+2 a^{2}\right)^{2}-4\left(k_{1}^{2}-a^{2}\right)\left(k_{2}^{2}-a^{2}\right)}} .
\end{aligned}
$$

Заметим, что $q_{1}^{ \pm}$является вещественным всегда, а $q_{2}^{ \pm}$- вещественно при выполнении условий $k_{1}^{2} \geqslant a^{2}, k_{2}^{2} \geqslant a^{2}$ или $k_{1}^{2} \leqslant a^{2}, k_{2}^{2} \leqslant a^{2}$.

Интерес представляет случай малых $a$, так как в задаче атомных столкновений $a$ пропорционально квадратному корню из отношения массы электрона к массе ядра. В этом случае асимптотика $q_{n}$ при $a \rightarrow 0$, которую можно легко получить из выражений (30) и (31), принимает вид

$$
q_{n}^{ \pm}= \pm k_{n} \pm \frac{1-(-1)^{n} 2\left(k_{1}^{2}+k_{2}^{2}\right) /\left(k_{1}^{2}-k_{2}^{2}\right)}{2 k_{n}} a^{2}+O\left(a^{4}\right), \quad n=1,2 .
$$

Эти формулы показывают, что в пределе $a \rightarrow 0$ величины $q_{n}$ стремятся к $k_{n}$. Важно отметить, что $q_{n}^{ \pm}-\left( \pm k_{n}\right)=O\left(a^{2}\right)$.

Систему уравнений (28) для двухкомпонентных векторов $\mathbf{C}^{n \pm}$ теперь можно решить путем подстановки значений $q_{n}^{ \pm}$в (28). В результате решение дается следующими выражениями:

$$
\begin{aligned}
& \mathbf{C}^{1 \pm}=\left[\begin{array}{c}
c_{1}^{1 \pm} \\
c_{2}^{1 \pm}
\end{array}\right]=N\left(a, q_{1}^{ \pm}, k_{1}\right)\left[\begin{array}{c}
1 \\
-\left(\left(q_{1}^{ \pm}\right)^{2}-k_{1}^{2}+a^{2}\right) / i 2 a q_{1}^{ \pm}
\end{array}\right]
\end{aligned}
$$

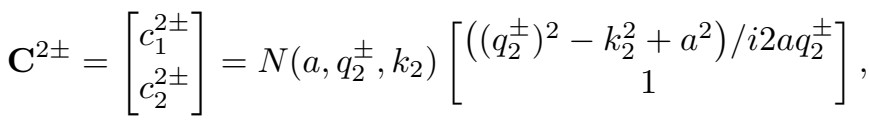

где нормировочные множители $N$ вычисляются по формуле

$$
N(a, q, k)=\left\{1+\frac{\left(q^{2}-k^{2}+a^{2}\right)^{2}}{4 a^{2} q^{2}}\right\}^{-1 / 2}
$$

с использованием подходящих значений для $q$ и $k$. Как и выше для $q_{n}^{ \pm}$, рассмотрим ситуацию, когда $a \rightarrow 0$. В этом случае получаем следующее поведение компонент 
векторов $\mathbf{C}^{n \pm}, n=1,2$ :

$$
c_{j}^{n \pm}=\delta_{j n} \pm i a \frac{2 k_{n}}{k_{1}^{2}-k_{2}^{2}}\left[1-\delta_{j n}\right]+O\left(a^{2}\right), \quad j=1,2 .
$$

Полученные четыре решения для уравнения (25)

$$
\boldsymbol{\Phi}^{n \pm}\left(r, q_{n}^{ \pm}\right)=\mathbf{C}^{n \pm} e^{i q_{n}^{ \pm} r}, \quad n=1,2,
$$

представляют собой набор асимптотических состояний, которые необходимы для построения асимптотики решения уравнений (22)

$$
\mathbf{F}(r) \sim \sum_{n=1}^{2} q_{n}^{-1 / 2}\left[b_{n}^{+} \boldsymbol{\Phi}^{n+}\left(r, q^{+}\right)+b_{n}^{-} \boldsymbol{\Phi}^{n-}\left(r, q_{n}^{-}\right)\right], \quad r \rightarrow \infty
$$

Аналогично определению (21), приведенному в предыдущем разделе, $(2 \times 2)$-матрица рассеяния вводится как матрица преобразования между амплитудой падающей волны $b_{j}^{-}$и амплитудой расходящейся волны $b_{j}^{+}$:

$$
(-1)^{\ell+1} b_{j}^{+}=\sum_{n=1}^{2} S_{j n}(a) b_{n}^{-}
$$

Асимптотическое представление (38) можно упростить, если использовать асимптотики (32) и (36) для $q_{n}^{ \pm}$и $\mathbf{C}^{n \pm}$ при $a \rightarrow 0$ в (37). Пренебрегая членами порядка $O\left(a^{2}\right)$, приходим к следующей форме компонент асимптотического решения (38):

$$
F_{j}(r) \sim \sum_{n=1}^{2} k_{n}^{-1 / 2}\left[b_{n}^{+} t_{j n}^{+} F_{n}^{+}\left(r, k_{n}\right)+b_{n}^{-} t_{j n}^{-} F_{n}^{-}\left(r, k_{n}\right)\right]
$$

Здесь коэффициенты $t_{j n}^{ \pm}$определены как

$$
t_{j n}^{ \pm}=\delta_{j n} \pm i a \frac{2 k_{n}}{k_{1}^{2}-k_{2}^{2}}\left[1-\delta_{j n}\right], \quad j, n=1,2 .
$$

Легко видеть, что формулы (40) и (41) совпадают с представлениями для асимптотических решений, полученных методом перепроецирования (см., например, формулы (18) и (21) работы [21]).

Следует заметить, что в пределе $a \rightarrow 0$ коэффициенты $t_{j n}^{ \pm}$принимают вид дельтасимволов Кронекера $\delta_{j n}$ и, соответственно, представления (40) превращаются в (20). Для матрицы рассеяния переход к этому пределу приводит к переходу $S_{j n}(a) \rightarrow S_{j n}$ с $S_{j n}$, определенной формулой (21).

Один из вариантов двухканальной модели (22) со специальным выбором параметров $a$ и $\epsilon_{j}, j=1,2$, рассматривался в работе [17], в которой анализ был сфокусирован на решении детерминантного уравнения (29). Наши аналитические результаты для асимптотических моментов (30), (31) в случае применения параметров упомянутой работы с очевидностью воспроизводят результаты работы [17]. 


\section{3. ОБЩИЙ СЛУЧАЙ МНОГОКАНАЛЬНЫХ УРАВНЕНИЙ}

В этом разделе мы рассматриваем общую ситуацию второго случая, описанного в п. 1.2 , когда $N$ состояний асимптотически остаются связанными при $r \rightarrow \infty$. В этой ситуации система уравнений (1) (или (16)) асимптотически расщепляется на две части: нетривиальную $(N \times N)$-систему для компонент $F_{j}, j=1, \ldots, N$, и тривиальную расцепленную систему уравнений вида (17) для компонент $F_{j}, j>N$. Последняя ведет к асимптотической форме компонент $F_{j}, j>N$, которая дана в соотношениях (20). Асимптотическую форму первого набора компонент для $j=1, \ldots, N$ следует строить с помощью решения соответствующей системы уравнений с нетривиальной асимптотической связью каналов. Как было аргументировано в п. 1.2, эта система получается из системы (1) или (16), если пренебречь всеми членами порядка $O\left(r^{-1-\delta}\right)$ и менее. Результирующая асимптотическая $(N \times N)$-система уравнений для вектор-функции $\boldsymbol{\Phi}(r)=\left\{\Phi_{1}(r), \ldots, \Phi_{N}(r)\right\}^{\mathrm{T}}$ в матричной форме имеет вид

$$
\left(-\mathbf{I} \frac{d^{2}}{d r^{2}}-2 \mathbf{A} \frac{d}{d r}-\mathbf{A}^{2}-\mathbf{K}^{2}\right) \boldsymbol{\Phi}(r)=0 .
$$

Здесь $(N \times N)$-матрицы $\mathbf{I}, \mathbf{K}^{2}$ и $\mathbf{A}$ даются своими матричными элементами

$$
[\mathbf{I}]_{j m}=\delta_{j m}, \quad\left[\mathbf{K}^{2}\right]_{j m}=\delta_{j m} k_{m}^{2}, \quad[\mathbf{A}]_{j m}=a_{j m} .
$$

Для решения уравнения (42) используем экспоненциальный анзац, который обычно применяется для решения дифференциальных уравнений и, в частности, использовался в работе [17] и в разделе 2 настоящей статьи.

Применение подстановки

$$
\boldsymbol{\Phi}(r)=\mathbf{C} e^{i q r}
$$

с неизвестной константой $q$ и вектором $\mathbf{C}=\left\{C_{1}, \ldots, C_{N}\right\}^{\mathrm{T}}$ сводит систему дифференциальных уравнений (42) к системе линейных алгебраических уравнений

$$
\left(q^{2} \mathbf{I}-2 q i \mathbf{A}-\mathbf{A}^{2}-\mathbf{K}^{2}\right) \mathbf{C}=0 .
$$

Нетривиальное решение (45) существует тогда и только тогда, когда определитель, который является полиномом степени $2 N$, равен нулю:

$$
D(q) \equiv \operatorname{det}\left(q^{2} \mathbf{I}-2 q i \mathbf{A}-\mathbf{A}^{2}-\mathbf{K}^{2}\right)=0 .
$$

В приложении показано, что уравнение (46) удовлетворяется только при вещественных $q$, когда выполняется условие $\left\langle\left(\mathbf{A}^{2}+\mathbf{K}^{2}\right) \mathbf{C}, \mathbf{C}\right\rangle>0$ для любого $\mathbf{C} \neq 0$, где $\langle\cdot, \cdot\rangle$ означает стандартное скалярное произведение в $\mathbb{C}^{N}$. Это условие в дальнейшем предполагается выполненным. Так как матрица $\mathbf{A}$ антисимметрична относительно транспонирования, $\mathbf{A}^{\mathrm{T}}=-\mathbf{A}$, свойство

$$
\left(q^{2} \mathbf{I}-2 q i \mathbf{A}-\mathbf{A}^{2}-\mathbf{K}^{2}\right)^{\mathrm{T}}=q^{2} \mathbf{I}+2 q i \mathbf{A}-\mathbf{A}^{2}-\mathbf{K}^{2}
$$

показывает, что левая часть (46) является четной функцией $q$, т. е. $D(-q)=D(q)$. Поэтому $2 N$ решений уравнения (46) образуют симметричное вещественное множество $\pm q_{1}, \ldots, \pm q_{N}$ такое, что $D\left( \pm q_{n}\right)=0, n=1, \ldots, N$. Для определенности 
знаки $q_{n}$ фиксируются так, чтобы $q_{n} \geqslant 0, n=1, \ldots, N$. Теперь $2 N$ векторов $\mathbf{C}^{n \pm}$, $n=1, \ldots, N$, можно найти как решения уравнений (45) при подстановке значений $\pm q_{n}$. Эти величины определяют $2 N$ решений уравнений (42) вида

$$
\boldsymbol{\Phi}^{n \pm}\left(r, q_{n}\right)=\mathbf{C}^{n \pm} e^{ \pm i q_{n} r} .
$$

Теперь мы имеем все необходимые составляющие для того, чтобы построить асимптотические граничные условия для уравнений (1). Компоненты $\Phi_{j}^{n \pm}$ состояний (48) для $j=1, \ldots, N$ вместе с компонентами $F_{j}^{ \pm}$из (18) для $j>N$ образуют базис для искомых асимптотических граничных условий для компонент $F_{j}(r)$ решений уравнений (1):

$$
\begin{aligned}
& F_{j}(r) \sim \sum_{n=1}^{N} q_{n}^{-1 / 2}\left[b_{n}^{-} \Phi_{j}^{n-}\left(r, q_{n}\right)+b_{n}^{+} \Phi_{j}^{n+}\left(r, q_{n}\right)\right], \quad j=1, \ldots, N, \\
& F_{j}(r) \sim k_{j}^{-1 / 2}\left[b_{j}^{-} F^{-}\left(r, k_{j}\right)+b_{j}^{+} F^{+}\left(r, k_{j}\right)\right], \quad j>N .
\end{aligned}
$$

Эти граничные условия являются нашим главным результатом, который завершает постановку задачи рассеяния для уравнений (1) в общей ситуации.

Соответствующая $S$-матрица теперь определяется как матрица преобразования между амплитудами падающей и расходящейся волн:

$$
(-1)^{\ell+1} b_{j}^{+}=\sum_{m \geqslant 1} S_{j m}(\mathbf{A}) b_{m}^{-} .
$$

Как и в предыдущем разделе, мы рассмотрим специальный случай, когда матрица связи каналов А является малой. Здесь удобно ввести скалярную константу связи $a$ таким образом: $\mathbf{A}=a \hat{\mathbf{A}}$, чтобы явно охарактеризовать порядок матрицы связи каналов. Для малых значений константы связи $a \rightarrow 0$ решение уравнения (45) можно получить по теории возмущений. Представим $\mathbf{C}$ и $q$ в виде

$$
q=q_{0}+a q_{1}+O\left(a^{2}\right), \quad \mathbf{C}=\mathbf{C}^{(0)}+a \mathbf{C}^{(1)}+O\left(a^{2}\right)
$$

и подставим в (45). В результате получим следующие уравнения для $\mathbf{C}^{(0)}$ и $\mathbf{C}^{(1)}$ :

$$
\begin{aligned}
\left(q_{0}^{2}-\mathbf{K}^{2}\right) \mathbf{C}^{(0)} & =0 \\
\left(q_{0}^{2}-\mathbf{K}^{2}\right) \mathbf{C}^{(1)} & =-2 q_{0}\left(q_{1}-i \hat{\mathbf{A}}\right) \mathbf{C}^{(0)}
\end{aligned}
$$

Первое уравнение (53) показывает, что $q_{0}^{2}$ и $\mathbf{C}^{(0)}$ должны быть собственным значением и собственным вектором диагональной матрицы $\mathbf{K}^{2}$ соответственно. Набором собственных значений $\mathbf{K}^{2}$ является множество $k_{1}^{2}, \ldots, k_{N}^{2}$, а соответствующие собственные векторы $\mathbf{C}^{(0) n}, n=1, \ldots, N$, даются компонентами $C_{j}^{(0) n}=\delta_{j n}$. Для решения второго уравнения (54) мы полагаем $\mathbf{C}^{(0)}=\mathbf{C}^{(0) n}$ и $q_{0}^{2}=k_{n}^{2}$, что ведет к равенству $q_{0}= \pm k_{n}$. Так как $\operatorname{Ker}\left(k_{n}^{2}-\mathbf{K}^{2}\right)$ является нетривиальным, требуется выполнение условия разрешимости

$$
\mp 2 k_{n}\left\langle\left(q_{1}-i \hat{\mathbf{A}}\right) \mathbf{C}^{(0) n}, \mathbf{C}^{(0) n}\right\rangle=0,
$$


для того чтобы гарантировать существование нетривиального решения уравнения (54). Вычисляя скалярное произведение, получаем $q_{1}=i \hat{a}_{n n}$, откуда следует, что $q_{1}=0$, так как $\hat{a}_{j n}$ является внедиагональной матрицей. Теперь уравнение (54) принимает форму

$$
\left(k_{n}^{2}-\mathbf{K}^{2}\right) \mathbf{C}^{(1) n \pm}= \pm 2 i k_{n} \hat{\mathbf{A}} \mathbf{C}^{(0) n} .
$$

Для компонент это дает выражение

$$
\left(k_{n}^{2}-k_{j}^{2}\right) C_{j}^{(1) n \pm}= \pm 2 i k_{n} \hat{a}_{j n}
$$

для $j \neq n$, в то время как

$$
C_{n}^{(1) n \pm}=0
$$

в соответствии со стандартным требованием теории возмущений [22]. Из уравнений (57), (58) видно, что выполняются равенства

$$
C_{j}^{(1) n \pm}= \pm \frac{2 i k_{n}}{k_{n}^{2}-k_{j}^{2}} \hat{a}_{j n}\left(1-\delta_{j n}\right) .
$$

Наконец, из (52) можно получить следующее выражение для $q$ и для компонент $\mathbf{C :}$

$$
\begin{gathered}
q= \pm k_{n}+O\left(a^{2}\right), \quad n=1, \ldots, N \\
C_{j}^{n \pm}=\delta_{j n} \pm \frac{2 i k_{n}}{k_{n}^{2}-k_{j}^{2}} a_{j n}\left(1-\delta_{j n}\right)+O\left(a^{2}\right), \quad j, n=1, \ldots, N .
\end{gathered}
$$

Здесь принято во внимание, что $a \hat{a}_{j n}=a_{j n}$. С этими представлениями явные выражения для главных членов компонент асимптотических состояний $\boldsymbol{\Phi}^{n \pm}$ даются следующими формулами:

$$
\Phi_{j}^{n \pm}\left(r, q_{n}\right)=t_{j n}^{ \pm} e^{ \pm i k_{n} r}+O\left(a^{2}\right)
$$

где $t_{j n}^{ \pm}$определяется как

$$
t_{j n}^{ \pm}=\delta_{j n} \pm \frac{2 i k_{n}}{k_{n}^{2}-k_{j}^{2}} a_{j n}\left(1-\delta_{j n}\right) .
$$

Соответствующие асимптотические при $r \rightarrow \infty$ граничные условия (49) для компонент $F_{j}(r)$ с $j=1, \ldots, N$ теперь принимают вид

$$
F_{j}(r) \sim \sum_{n=1}^{N} k_{n}^{-1 / 2}\left[b_{n}^{-} t_{j n}^{-} e^{-i k_{n} r}+b_{n}^{+} t_{j n}^{+} e^{i k_{n} r}\right]+O\left(a^{2}\right) .
$$

Заметим, что последняя формула совпадает с формулой (18), которая получена в работе [21] методом перепроецирования. Компоненты матрицы $t$ в (63) также идентичны соответствующим компонентам $t$ матрицы из работы [21], если принять во внимание определения (9), (13) и (19).

Выведенные формулы (60) и (61) дают старший порядок по отношению к константе связи $a$ для $q$ и $\mathbf{C}$. Следующие по порядку величины члены разложения можно получить в случае необходимости с помощью стандартной теории возмущений [22]. Следует также упомянуть, что случай вырожденных асимптотических каналов рассеяния можно рассмотреть с помощью соответствующего варианта теории возмущений, в связи с чем может потребоваться применение дополнительных коррекций по константе связи при построении асимптотических граничных условий (как, например, показано в статье [23]). 


\section{4. ЗАКЛЮЧЕНИЕ}

Мы представили общий формализм построения асимптотических граничных условий для решения адиабатической многоканальной задачи рассеяния в случае, когда матрица неадиабатической связи каналов нетривиальна на больших межъядерных расстояниях. Эти асимптотические условия обобщают форму Борна-Оппенгеймера для асимптотических граничных условий, которые обычно используются в случае асимптотического расцепления уравнений. Ненулевая асимптотика матричных элементов матрицы неадиабатической связи каналов является фундаментальным свойством подхода Борна-Оппенгеймера, поэтому построение асимптотических решений для многоканальных уравнений с такой связью каналов является важным не только с точки зрения общей теории, но и с практической точки зрения при вычислении сечений неупругих процессов. Вычисление сечений неупругих столкновений с ненулевой асимптотикой матрицы неадиабатической связи каналов успешно выполнено с помощью метода перепроецирования для ряда процессов столкновений, например, столкновений в системах $\mathrm{Li}+\mathrm{Na}$ [12], $\mathrm{He}+\mathrm{H}[23], \mathrm{Mg}+\mathrm{H}$ [24], $\mathrm{Li}^{+}+\mathrm{He}$ и $\mathrm{Li}+\mathrm{He}^{+}[25]$.

Асимптотические решения (63), (64), выведенные в рамках общего формализма настоящей работы, совпадают в старшем порядке теории возмущений с асимптотическими решениями, использованными в методе перепроецирования [9], [10], [12], [21]. По этой причине вычисление сечений неупругих процессов дает идентичный результат. Таким образом, эти вычисления предоставляют практическое подтверждение для формализма данной работы.

Формулировка многоканальной задачи рассеяния с полученными в данной работе условиями охватывает два наиболее важных случая короткодействующего поведения и дальнодействующего постоянного асимптотического поведения матрицы неадиабатической связи каналов. Случай медленного типа $O\left(r^{-1}\right)$ поведения матрицы неадиабатической связи каналов и/или адиабатических потенциалов также представляет интерес (в особенности для гиперсферического адиабатического подхода [4]). Решение задачи нахождения асимптотических граничных условий в этом случае требует использования дополнительной специальной техники и будет дано в другой работе.

Выведенная асимптотическая форма для решения адиабатических связанных уравнений открывает путь для использования недавно разработанного подхода расщепления потенциала для решения многоканальной задачи рассеяния как в диабатическом, так и в адиабатическом представлении многоканальных уравнений [26], [27].

\section{ПРИЛОЖЕНИЕ}

Покажем, что уравнение (46) при условии, что $\left\langle\left(\mathbf{A}^{2}+\mathbf{K}^{2}\right) \mathbf{C}, \mathbf{C}\right\rangle>0$ для любого $\mathbf{C} \neq 0$, допускает нетривиальные решения только для вещественных значений параметра $q$, т. е. $\operatorname{Ker}\left(q^{2} \mathbf{I}-2 q i \mathbf{A}-\mathbf{A}^{2}-\mathbf{K}^{2}\right)$ нетривиально только для вещественных $q$. Пусть $q$ комплексно и $\mathbf{C} \in \operatorname{Ker}\left(q^{2} \mathbf{I}-2 q i \mathbf{A}-\mathbf{A}^{2}-\mathbf{K}^{2}\right)$, тогда

$$
\left\langle\left[q^{2} \mathbf{I}-2 q i \mathbf{A}-\mathbf{A}^{2}-\mathbf{K}^{2}\right] \mathbf{C}, \mathbf{C}\right\rangle=0 .
$$


Представим $q$ как $q=\alpha+i \beta$ и, воспользовавшись тем, что матрица $i \mathbf{A}$ эрмитова и тем самым $\langle i \mathbf{A C}, \mathbf{C}\rangle$ вещественно, отделим в (65) вещественную и мнимую части:

$$
\begin{aligned}
\left(\alpha^{2}-\beta^{2}\right)\langle\mathbf{C}, \mathbf{C}\rangle-2 \alpha\langle i \mathbf{A C}, \mathbf{C}\rangle-\left\langle\left(\mathbf{A}^{2}+\mathbf{K}^{2}\right) \mathbf{C}, \mathbf{C}\right\rangle & =0 \\
2 \beta[\alpha\langle\mathbf{C}, \mathbf{C}\rangle-\langle i \mathbf{A C}, \mathbf{C}\rangle] & =0 .
\end{aligned}
$$

При $\beta \neq 0$ из (67) следует равенство

$$
\alpha\langle\mathbf{C}, \mathbf{C}\rangle-\langle i \mathbf{A C}, \mathbf{C}\rangle=0 .
$$

Равенство (68) сводит уравнение (66) к виду

$$
\left(\alpha^{2}+\beta^{2}\right)\langle\mathbf{C}, \mathbf{C}\rangle+\left\langle\left(\mathbf{A}^{2}+\mathbf{K}^{2}\right) \mathbf{C}, \mathbf{C}\right\rangle=0 .
$$

$\mathrm{C}$ другой стороны, при $\mathbf{C} \neq 0$ из неравенства $\langle\mathbf{C}, \mathbf{C}\rangle>0$, которое выполняется по определению скалярного произведения, и неравенства $\left\langle\left(\mathbf{A}^{2}+\mathbf{K}^{2}\right) \mathbf{C}, \mathbf{C}\right\rangle>0$, которое выполняется по предположению, следует неравенство

$$
\left(\alpha^{2}+\beta^{2}\right)\langle\mathbf{C}, \mathbf{C}\rangle+\left\langle\left(\mathbf{A}^{2}+\mathbf{K}^{2}\right) \mathbf{C}, \mathbf{C}\right\rangle \geqslant \beta^{2}\langle\mathbf{C}, \mathbf{C}\rangle
$$

для любого $\alpha$. При $\beta \neq 0$ из этого неравенства получается, что уравнение (69) выполняется только при $\mathbf{C}=0$. Тем самым мы показали, что при выполнении условия $\left\langle\left(\mathbf{A}^{2}+\mathbf{K}^{2}\right) \mathbf{C}, \mathbf{C}\right\rangle>0$ для матриц $\mathbf{A}^{2}$ и $\mathbf{K}^{2}$ параметр $q$, при котором множество $\operatorname{Ker}\left(q^{2} \mathbf{I}-2 q i \mathbf{A}-\mathbf{A}^{2}-\mathbf{K}^{2}\right)$ содержит нетривиальные векторы, может быть только вещественным.

\section{Список литературы}

[1] N. F. Mott, H.S. W. Massey, The Theory of Atomic Collisions, Clarendon, Oxford, 1949.

[2] M. Born, R. Oppenheimer, "Zur Quantentheorie der Molekeln", Ann. Phys., 389:20 (1927), $457-484$.

[3] C. A. Mead, D. G. Truhlar, "Conditions for the definition of a strictly diabatic electronic basis for molecular systems", J. Chem. Phys., 77:12 (1982), 6090-6098.

[4] B. D. Esry, H. R. Sadeghpour, "Split diabatic representation", Phys. Rev. A, 68:4 (2003), 042706, 8 pp.

[5] J. B. Delos, W. R. Thorson, "Diabatic and adiabatic representations for atomic collision processes", J. Chem. Phys., 70:4 (1979), 1774-1790.

[6] J. B. Delos, "Theory of electronic transitions in slow atomic collisions", Rev. Modern Phys., 53:2 (1981), 287-357.

[7] M. Gargaud, J. Hanssen, R. McCarroll, P. Valiron, "Charge exchange with multiply charged ions at low energies: application to the $\mathrm{N}^{3+} / \mathrm{H}$ and $\mathrm{C}^{4+} / \mathrm{H}$ systems", J. Phys. B, 14:13 (1981), 2259-2276.

[8] A. Macias, A. Riera, "Ab initio quantum chemistry in the molecular model of atomic collisions", Phys. Rep., 90:5 (1982), 299-376.

[9] J. Grosser, T. Menzel, A. K. Belyaev, "Approach to electron translation in low-energy atomic collisions", Phys. Rev. A, 59:2 (1999), 1309-1316.

[10] A. K. Belyaev, D. Egorova, J. Grosser, T. Menzel, "Electron translation and asymptotic couplings in low-energy atomic collisions", Phys. Rev. A, 64:5 (2001), 052701, 9 pp.

[11] A. K. Belyaev, A. Dalgarno, R. McCarroll, "The dependence of nonadiabatic couplings on the origin of electron coordinates", J. Chem. Phys., 116:13 (2002), 5395-5400. 
[12] A. K. Belyaev, "Revised Born-Oppenheimer approach and a reprojection method for inelastic collisions", Phys. Rev. A, 82:6 (2010), 060701, 4 pp.

[13] L. I. Ponomarev, S. I. Vinitsky, J. Phys. B, 12 (1979), 567.

[14] L.I. Ponomarev, S. I. Vinitsky, F.R. Vukajlovic, "Adiabatic representation in the three-body problem with the Coulomb interaction. II. The effective two-level approximation", J. Phys. B, 13:5 (1980), 847-868.

[15] L. I. Ponomarev, L. N. Somov, F. R. Vukajlovic, "Adiabatic representation in the three-body problem with a Coulomb interaction. III. Asymptotics of solutions as $R \rightarrow \infty$ ", J. Phys. B, 14:4 (1981), 591-602.

[16] L. Bracci, C. Chiccoli, P. Pasini, G. Fiorentini, V.S. Melezhik, J. Wożniak, "About the boundary conditions for the three-body scattering problem in the adiabatic representation", Il Nuovo Cimento, 105:4 (1990), 459-468.

[17] V.I. Korobov, "Multichannel scattering with velocity-dependent asymptotic potentials", J. Phys. B, 27:4 (1994), 733-746.

[18] A. Adamczak, M. P. Faifman, L. I. Ponomarev, V. I. Korobov, V. S. Melezhik, R. T. Siegel, J. Wożniak, "Atlas of cross sections for scattering of muonic hydrogen atoms on hydrogen isotope molecules", Atomic Data and Nuclear Data Tables, 62:2 (1996), 255-344.

[19] С. П. Меркурьев, С. Л. Яковлев, "Квантовая теория рассеяния для $N$ тел в конфигурационном пространстве", ТМФ, 56:1 (1983), 60-73.

[20] R. G. Newton, Scattering Theory of Waves and Particles, Springer, New York, 1982.

[21] A. K. Belyaev, "Nonadiabatic effects in inelastic collisional processes", Phys. Scr., 80:4 (2009), 048113, 7 pp.

[22] Л. Д. Ландау, Е. М. Лифшиц, Теоретическая физика, т. 3: Квантовая механика. Нерелятивистская теория, Наука, М., 1974.

[23] A. K. Belyaev, "Excitation cross sections in low-energy hydrogen-helium collisions", Phys. Rev. A, 91:6 (2015), 062709, 4 pp.

[24] A. K. Belyaev, P. S. Barklem, A. Spielfiedel, M. Guitou, N. Feautrier, D. S. Rodionov, D. V. Vlasov, "Cross sections for low-energy inelastic $\mathrm{Mg}+\mathrm{H}$ and $\mathrm{Mg}^{+}+\mathrm{H}^{-}$collisions", Phys. Rev. A, 85:3 (2012), 032704, 9 pp.

[25] A. K. Belyaev, D. S. Rodionov, L. Augustovičová, P. Soldán, W. P. Kraemer, "Full quantum study of non-radiative inelastic processes in lithium-helium ion-atom collisions", Mont. Not. Roy. Astron. Soc., 449:3 (2015), 3323-3332.

[26] M. V. Volkov, S. L. Yakovlev, E. A. Yarevsky, N. Elander, "Potential splitting approach to multichannel Coulomb scattering: the driven Schrödinger equation formulation", Phys. Rev. A, 83:3 (2011), 032722, 12 pp.

[27] M. V. Volkov, S. L. Yakovlev, E. A. Yarevsky, N. Elander, "Adiabatic versus diabatic approach to multichannel Coulomb scattering for mutual neutralisation reaction $\mathrm{H}^{+}+\mathrm{H}^{-} \rightarrow$ $\mathrm{H}_{2}^{*} \rightarrow \mathrm{H}(1)+\mathrm{H}(n) "$, Chem. Phys., 462 (2015), 57-64.

Поступила в редакцию 24.08.2017, после доработки 9.11.2017 